\title{
Simple Molecular Absorption Loss Model for 200-450 Gigahertz Frequency Band
}

\author{
Joonas Kokkoniemi, Janne Lehtomäki, and Markku Juntti \\ Centre for Wireless Communications (CWC), \\ University of Oulu, P.O. Box 4500, 90014 Oulu, Finland \\ Email: joonas.kokkoniemi@oulu.fi
}

\begin{abstract}
This paper derives a simplified polynomial molecular absorption loss model for $200-450 \mathrm{GHz}$ band. This band has a high potential for near future short range, high data rate applications due to large spectral resources and reasonable path loss. The frequencies around $300 \mathrm{GHz}$ are among the first ones where the molecular absorption loss becomes a significant factor. This loss increases exponentially with the distance and is not a major issue at short distances (few meters), but entirely blocks signals over large distances. Modeling of the molecular absorption loss is relatively straightforward, but requires large numbers of parameters from spectroscopic databases. This paper derives a simplified polynomial absorption loss model for the major absorption lines. This extends and gives more accurate absorption loss values in comparison to the existing ITU-R absorption loss models. The simplified polynomial expressions by ITU-R are mostly limited to about $350 \mathrm{GHz}$ frequency, although being rather accurate up to about $400 \mathrm{GHz}$. This paper gives a simpler and more accurate absorption loss model to those bands. A part of the considered band, 275-450 $\mathbf{G H z}$ band, is subject to World Radiocommunication Conference 2019 (WRC-19) for allocation and operational characterization for the future communication applications.
\end{abstract}

\section{INTRODUCTION}

The millimeter wave frequencies (mmWave, 30-300 GHz) are very interesting for the future $5 \mathrm{G}$ and beyond wireless communications systems and applications [1]. These frequencies offer very large bandwidths and are mainly suitable for short range communications. However, larger link distances are possible with large antenna gains, giving opportunity to utilize these frequencies, e.g., in backhaul applications. For instance, an 850 meter link has been shown to be feasible at $240 \mathrm{GHz}$ frequency [2]. The low $\mathrm{THz}$ band (300-1000 $\mathrm{GHz}$ ) is the next frontier after the mmWave frequencies have been conquered. There is no fundamental cut-off at $300 \mathrm{GHz}$, but the utilization of the low $\mathrm{THz}$ band is mainly up to development of THz-capable devices.

One of the key things in designing novel systems is knowing the channel. It sets the fundamental limits to the communication performance and understanding those limits helps in maximizing the physical layer performance. In this paper, we consider a frequency band between the mmWave and $\mathrm{THz}$ bands, 200-450 GHz band. A part of this band (275$450 \mathrm{GHz}$ ) is in the agenda for allocation and technological mapping in the World Radiocommunication Conference 2019 (WRC-2019). The considered band also introduces some of the first major molecular absorption frequencies [3]. The molecular absorption is caused by the fact that the energy of the photons is absorbed by the molecules in the medium [4]. The significance of this loss mechanism is not high around 300 $\mathrm{GHz}$ frequencies or at low distances due to the dominant free space path loss (FSPL). However, there are some significant absorption lines at 325,380 , and 450 that dominate the overall path loss at long distances. The molecular absorption loss increases exponentially with the distance according to the BeerLambert law [4], and, therefore, its impact with respect to the FSPL (square-law) also depends on the distance. Modeling the molecular absorption loss requires spectroscopic databases to calculate all the properties of the molecules. The one used here is the HITRAN database [5]. The main objective of this paper is to introduce a compact parametric molecular absorption model that omits the usage of the spectroscopic databases, and, thereby, makes it easier to estimate the absorption loss with high accuracy.

ITU-R has presented the model for calculation of gaseous attenuation up to $1000 \mathrm{GHz}$ in ITU-R P.676-8 [6]. The model in [6] is line-by-line based and the results from it correspond to those obtained by using the HITRAN database. However, ITU-R uses a modified full Lorentz line shape that is not in general recommended for the millimeter frequencies [7]. Instead, a van Vleck-Weisskopf, or van Vleck-Huber line shapes are utilized for those frequencies [7] as in the case of proposed model herein. Furthermore, the full model by ITU-R is not suitable for analytical calculations or for a quick use, since it requires using a significant number (553) of tabulated parameters and complicated functions. In [6], a polynomial based approximation has also been presented. It is valid up to $350 \mathrm{GHz}$, but is reasonably accurate up to about $450 \mathrm{GHz}$. It should be noticed that a newer version ITU-R 676-11 also exists, but that version does not have a polynomial model. We use the older version, since the polynomial model therein is related to our work in this paper.

However, compared to our models, the models in [6] have several weaknesses. First of all, even though [6] includes lines even at $1780 \mathrm{GHz}$, it is only specified to be valid for frequencies up to $350 \mathrm{GHz}$. The simplified model in the newer version is also limited to $350 \mathrm{GHz}$. The model also includes nine terms. If some of those are removed, they may also affect frequencies in different bands. For example, the term involving $1780 \mathrm{GHz}$ needs to kept or the level of the attenuation between the peaks at lower frequencies is way off. In general, the ITU- 
$\mathrm{R}$ models are relatively accurate below $450 \mathrm{GHz}$. However, they overestimate the wing absorption, i.e., the loss far away from the center of the absorption line. In this paper, we will extend the frequency range, give more accurate estimate for the absorption loss, and simplify the estimation for it. Our model utilizes five polynomials that can be reduced, if one is interested in frequencies below $400 \mathrm{GHz}$.

We have derived a simplified molecular absorption loss model in the past [3]. It was intended for the 275-400 $\mathrm{GHz}_{\text {band }}{ }^{1}$. The model presented in this paper extends this frequency band with a cost of slightly increased complexity (three polynomials versus five in the model herein). However, the model given here can be reduced from five polynomials into two or three polynomials, if one is interested in the below $400 \mathrm{GHz}$ frequencies only. The proposed model is based on the Lorentz absorption line shape. The response given by the Lorentz model overestimates the wing absorption. The correct line shape, given by the van Vleck-Huber line shape, is estimated based on a fitting function. In our previous paper [3], this fitting function was partly incorrect as it did not take into account the variations in the wing absorption based on the humidity. This has been fixed for the proposed model in this paper. The accuracy of the proposed model is shown to be very good in the numerical results. We also show that ITU-R models give somewhat pessimistic image of the channel in the long range applications.

The rest of this paper is organized as follows. Section II gives the derived proposed polynomial absorption loss model, Section III gives some numerical examples, and Section IV concludes the paper.

\section{Simplified Molecular AbSorption Loss Model}

The molecular absorption is given by the Beer-Lambert law. It describes the transmittance, i.e., the amount of power that is capable of propagating through the channel. The transmittance depends on the link distance and absorption coefficient by [4], [7]

$$
\tau(f, r)=\frac{P_{r}(f)}{P_{t}(f)}=e^{-\Sigma_{j} \kappa_{a}^{j}(f) r},
$$

where $\tau(f, r)$ is the transmittance, $f$ is the frequency, $r$ is the distance from transmitter (Tx) to receiver $(\mathrm{Rx}), P_{t}(f)$ and $P_{r}(f)$ are Tx and Rx power, respectively, and $\kappa_{a}^{j}(f)$ is the absorption coefficient of the $j$ th absorbing species at frequency $f$. The absorption coefficient can be calculated with the help of databases, such as the HITRAN database [5] as it will be shown below. The details of the calculation of the absorption coefficient can be found, e.g., in our previous paper about simplified channel model [3].

The parametric polynomial model for the molecular absorption loss is derived based on the properties of the individual absorption lines. If we know the loss coefficient $y_{i}$ at absorption

\footnotetext{
${ }^{1}$ It should be noted that the model in [3] had a mistake in the polynomials where the speed of light should have been multiplied by one hundred. In that paper, the speed of light was divided by one hundred. The error was only present in the paper and not in the computer implementation. The error has been fixed for the model in this paper
}

line $i$, we can present the path loss model as The molecular absorption loss is given by the Beer-Lambert law [4], [7]

$$
\operatorname{PL}_{\text {abs }}(f, \mu)=e^{d\left(\sum_{i} y_{i}(f, \mu)+g(f, \mu)\right)},
$$

where $f$ is the desired frequency grid, $y_{i}$ is an absorption coefficient for the $i$ th absorption line in the studied band, $\mu$ is the volume mixing ratio of water vapor, which can be estimated from the relative humidity, e.g., similarly as we did in [3], and $g(f, \mu)$ is a fit polynomial to rectify errors in the model. Those are explained in more detail below. Notice that we assume that water vapor dominates the molecular absorption loss and other molecules are not modeled. This is a fair assumption as all the major absorption lines at 200 $450 \mathrm{GHz}$ band are caused by water vapor. The validity of this assumption is further shown in the numerical results.

The polynomials for the major absorption lines at the 200 $450 \mathrm{GHz}$ band are as follows:

$$
\begin{aligned}
y_{1}(f, \mu) & =\frac{A(\mu)}{B(\mu)+\left(\frac{f}{100 c}-p_{1}\right)^{2}}, \\
y_{2}(f, \mu) & =\frac{C(\mu)}{D(\mu)+\left(\frac{f}{100 c}-p_{2}\right)^{2}}, \\
y_{3}(f, \mu) & =\frac{E(\mu)}{F(\mu)+\left(\frac{f}{100 c}-p_{3}\right)^{2}}, \\
y_{4}(f, \mu) & =\frac{G(\mu)}{H(\mu)+\left(\frac{f}{100 c}-p_{4}\right)^{2}}, \\
g(f, \mu)=\frac{\mu}{0.0157}\left(q_{1} f^{4}+q_{2} f^{3}+q_{3} f^{2}+q_{4} f\right. &
\end{aligned}
$$

where the frequency $f$ is given in Hertz, and

$$
\begin{gathered}
A(\mu)=0.2251 \mu(0.1314 \mu+0.0297), \\
B(\mu)=(0.4127 \mu+0.0932)^{2}, \\
C(\mu)=2.053 \mu(0.1717 \mu+0.0306), \\
D(\mu)=(0.5394 \mu+0.0961)^{2}, \\
E(\mu)=0.177 \mu(0.0832 \mu+0.0213), \\
F(\mu)=(0.2615 \mu+0.0668)^{2}, \\
G(\mu)=2.146 \mu(0.1206 \mu+0.0277), \\
H(\mu)=(0.3789 \mu+0.0871)^{2},
\end{gathered}
$$

and with $p_{1}=10.84 \mathrm{~cm}^{-1}, p_{2}=12.68 \mathrm{~cm}^{-1}, p_{3}=14.65$ $\mathrm{cm}^{-1}, p_{4}=14.94 \mathrm{~cm}^{-1}, q_{1}=8.495 \times 10^{-48}, q_{2}=$ $-9.932 \times 10^{-36}, q_{3}=4.336 \times 10^{-24}, q_{4}=-8.33 \times 10^{-13}$, and $q_{5}=5.953 \times 10^{-2}$. The absorption lines $y_{1}, y_{2}, y_{3}$, and $y_{4}$ correspond to strong absorption lines at center frequencies $325 \mathrm{GHz}, 380 \mathrm{GHz}, 439 \mathrm{GHz}$, and $448 \mathrm{GHz}$, respectively.

The derived model above is based on the Lorentz line shape due to it has a simpler representation than the van VleckHuber line shape. The model above is obtained by utilizing 


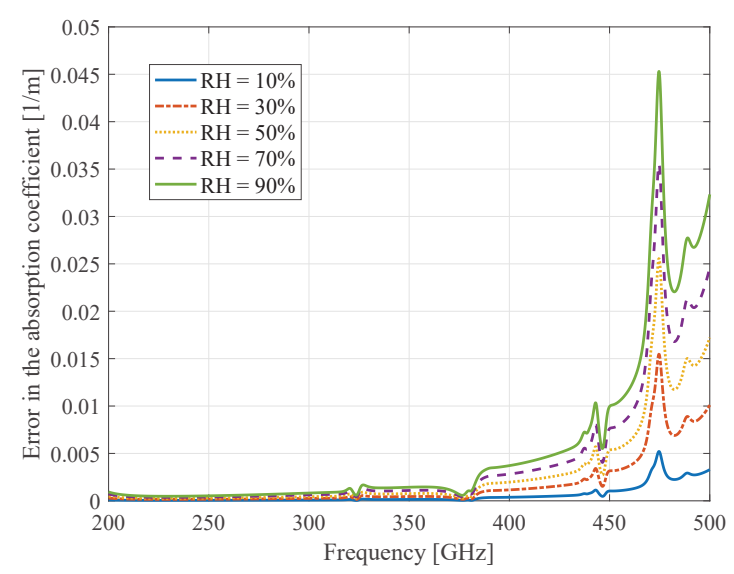

Fig. 1. An error to the exact response as a function of the frequency for different humidity levels before applying the fitting term $g(f, \mu)$.

the line-specific data from HITRAN and by leaving only the humidity (with pressure and temperature dependencies) and frequency grid floating. The difference between the Lorentz and van Vleck-Huber line shapes is rectified by the fit polynomial $g(f, \mu)$. This term is obtained by curve fitting on the error between the theoretical exact response to the output of the polynomial model. It would be possible to calculate the exact difference theoretically, but this only applies to the inband absorption and this approach would not consider the outof-band wing absorption. Therefore, it is easier and to some extent more accurate to just curve fit the error in order to produce the numerical fit polynomial $g(f, \mu)$.

The Lorentz line shape overestimates the wing absorption per line. On the other hand, by just taking into account the absorption line characteristics of the strongest in-band lines, the derived model underestimates the far wing absorption from the lines outside the considered band. The fit polynomial $g(f, \mu)$ rectifies these issues with good accuracy. Furthermore, the far wing absorption also depends on the amount of water vapor in the air. This causes error in the fit. Fig. 1 shows the error of the derived summed absorption coefficient as a function of frequency for various water vapor levels at 25 degrees centigrade. The humidity levels correspond to $\mu=$ [ $\left.\begin{array}{lllll}0.0031 & 0.0094 & 0.0157 & 0.0220 & 0.0282\end{array}\right]$ for $\mathrm{RH}=\left[\begin{array}{lll}10 \% & 30 \%\end{array}\right.$ $50 \% 70 \% 90 \%$ ], respectively. We can see that the error is linear with respect to the volume mixing ratio of water vapor. Therefore, the fit polynomial $g(f, \mu)$ was included with a term $\mu / 0.0157$, in which 0.0157 is the design reference volume mixing ratio of water vapor. Since this model only depends on the amount of water vapor in the air, the temperature dependence mainly contributes to the volume mixing ratio of water vapor by the saturation water vapor pressure. The line intensity $S$ is usually scaled for temperatures outside the reference temperature (296 K for the HITRAN database), but not taking this into account only produces a small error and most of its impact is included in the fit polynomial $g(f, \mu)$. The error in the proposed model as well as comparison of the

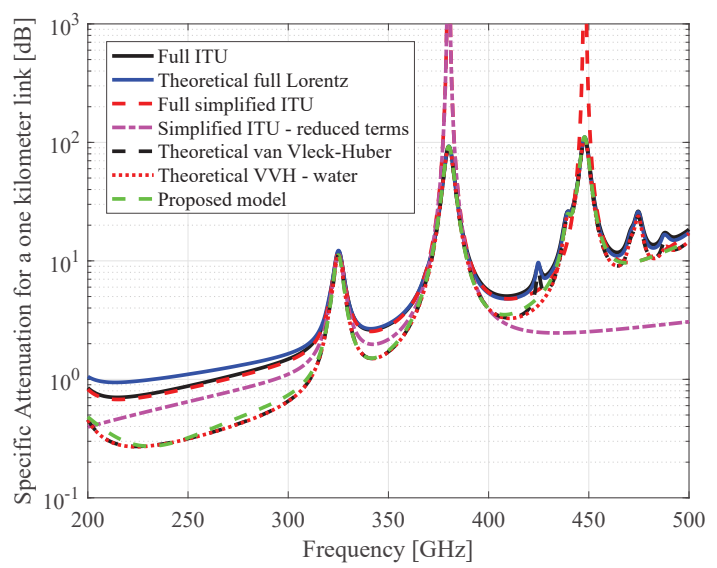

Fig. 2. Molecular absorption loss at $1 \mathrm{~km}$ distance at 25 degrees centigrade and $10 \%$ relative humidity $(\mu=0.0031)$.

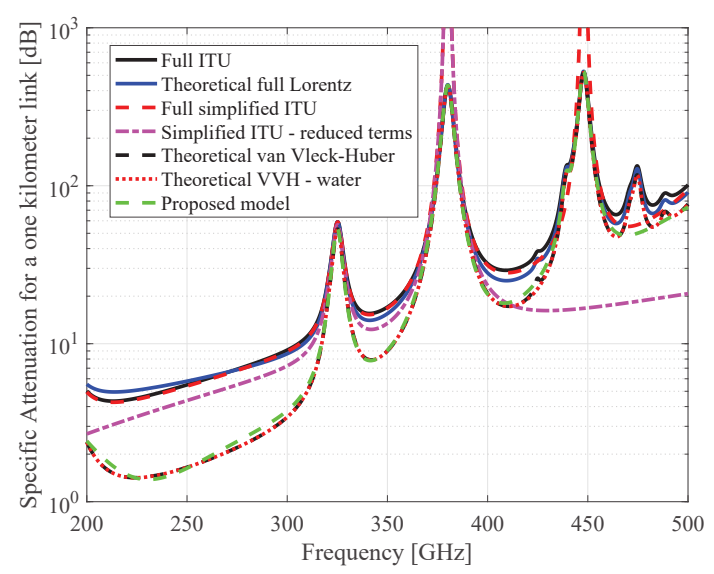

Fig. 3. Molecular absorption loss at $1 \mathrm{~km}$ distance at 25 degrees centigrade and $50 \%$ relative humidity $(\mu=0.0157)$.

error to ITU-R models is given in the numerical results.

\section{NUMERICAL RESULTS}

In this section, we present some numerical examples on the derived molecular absorption loss model and compare those to the corresponding ITU-R models and the exact response. We also show the error values of different models. All the results are given at 25 degrees centigrade and variable humidity levels. The absolute temperature is not that important here, since the amount of water vapor in the air is the most important factor in the absorption loss. However, this depends on the temperature and pressure, but those mainly set the upper bound for the volume mixing ratio of water in the air via saturation volume mixing ratio of water in the air.

Figs. 2-4 show the performance of different models. The Full ITU model is a full spectroscopic model similar to the exact theoretical models presented in this paper. It uses the modified full Lorentz line shape function and it gives in practice nearly identical response to the full Lorentz model with slightly less wing absorption. The full simplified ITU 


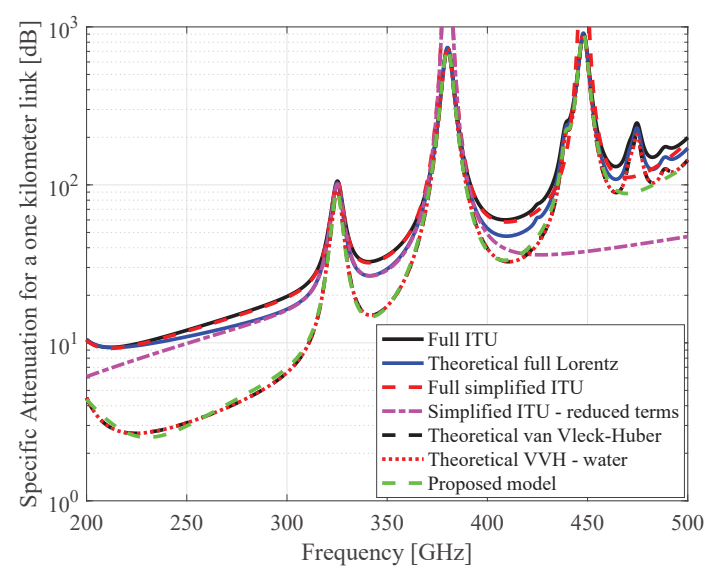

Fig. 4. Molecular absorption loss at $1 \mathrm{~km}$ distance at 25 degrees centigrade and $90 \%$ relative humidity $(\mu=0.0282)$.

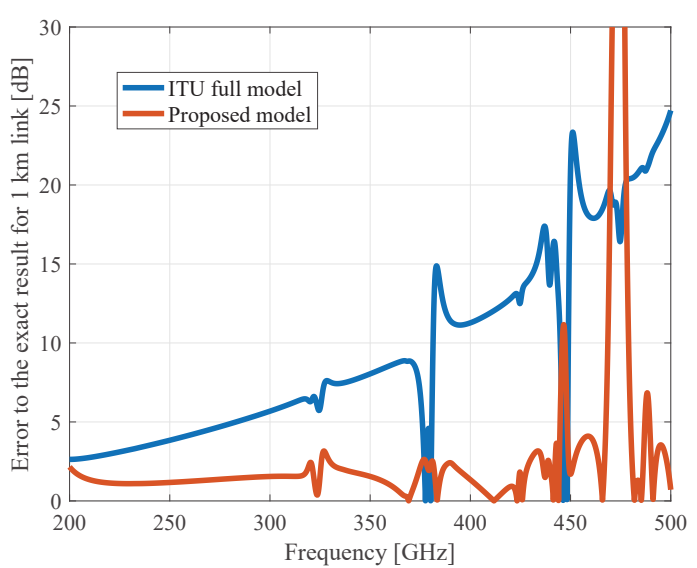

Fig. 5. Absolute errors given by the ITU-R full model and the proposed model to the exact theory for a one kilometer link.

model and its reduced version are polynomial based simplified solutions, such as the ones given in this paper. The theoretical van Vleck-Huber model is the correct one for these frequencies and used as a reference in this section. We have also calculated the pure water vapor impact mainly to show that the validity of utilizing just the water vapor lines in the absorption coefficient estimation. The response is practically identical to the full model with all the molecules. Finally, the green dashed line gives the output of the proposed model.

Figs. 2-4 are given for a distance of 1000 meters to show the behavior of the models in an extreme case. The figures show that the ITU-R models do very well with the assumption of the full Lorentz model. The simplified models overestimate the absorption loss at the peak of the absorption lines. This is not absolutely crucial with long distances as the absorption kills the signals in any case. However, at lower distance where the absorption is not significant, this overestimation may give too pessimistic image of the channel. It should be remembered that the ITU-R simplified models are not intended to be used above $350 \mathrm{GHz}$, although they are relatively accurate even above this limit.

The ITU-R models do overestimate the wing absorption that translates into too high absorption in the transmission windows, that is, where the absorption loss is low. The accurate model gives absorption losses of few decibels to about ten $\mathrm{dB}$ lower values. Especially, in the long range applications this is significant in the link budget calculations. The significance of the molecular absorption loss is considered below in more detail. The below figures also show that our model describes the theoretical absorption response very accurately within few $\mathrm{dB}$ error at most in the considered band. This is more clearly visible in Fig. 5 where the absolute errors of the ITU-R full model and the proposed model are compared at 25 degrees centigrade and $50 \%$ relative humidity. The error in the full Lorentz model increases as a function of frequency. Our model does have some error in it, but it is confined into roughly $\pm 2 \mathrm{~dB}$ region. The error in both cases is mostly visible in the locations of the strong absorption peaks. As stated above, those errors are not that significant due to strong absorption will kill the signal in any case. Furthermore, the error exponentially decreases with the overall absorption loss with decreasing distance, and thus, the error is at highest at extreme distances. Since the one kilometer links are most definitely at the higher end of the possible applications for mmWave and $\mathrm{THz}$ frequencies, the error in the most cases will be much smaller. The relative error between the ITU-R models and our model also increases as the humidity increases due to amount of water vapor in the atmosphere scales the absorption loss as it is the main contributor for the loss. That is, when there is no water vapor in the air, the absorption loss is insignificant according to all the models herein. When the water vapor partial pressure is saturated, the absorption loss is at its peak at given atmospheric conditions, and therefore, also the error is the highest.

In [3] we showed a simplified model up to $400 \mathrm{GHz}$. This model had some issues as detailed above. Those issues have been fixed for this paper, but the model herein is also more complicated with five polynomials. With more sophisticated fitting parameter $g(f, \mu)$, we can estimate the wing absorption even if we reduce the terms. That is, if one is interested on the absorption loss below $400 \mathrm{GHz}$ frequencies, one can utilize only the $y_{1}(f, \mu)+g(f, \mu)$ or $y_{1}(f, \mu)+y_{2}(f, \mu)+g(f, \mu)$ to estimate the absorption loss up to $330 \mathrm{GHz}$ or $390 \mathrm{GHz}$ respectively. Fig. 6 shows the responses of these reduced terms and compares those to the exact theory. We can see that the reduced terms give a very good estimate up to the above specified limit frequencies (notice again the irrelevance of a small error in peak absorption case). Therefore, the model presented herein can take very simple form and still reliably estimate the absorption loss if one is interested to estimate the absorption loss up to the above mentioned frequency limits. For instance, if one performs simulations at $275-$ $325 \mathrm{GHz}$ band, the two polynomial representation is simple to implement and accurate for the considered band.

Finally, let us discuss about the significance of the absorption loss at different link distances. The FSPL is always 


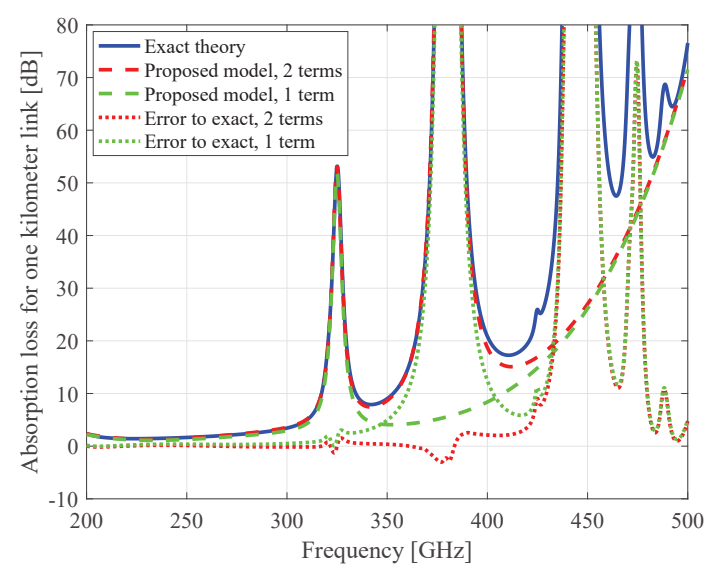

Fig. 6. Reduced versions of the proposed model giving absorption losses up to about $330 \mathrm{GHz}$ (1 term) and $390 \mathrm{GHz}$ (2 terms).

present and usually contributes to large portion of the loss experienced by the signals in the channel (neglecting the nonLOS phenomena). The free space path loss is given by the familiar equation:

$$
\operatorname{PL}_{\mathrm{FSPL}}(r, f)=\frac{(4 \pi r f)^{2}}{c^{2}},
$$

where $c$ is the speed of light. Assuming no other loss mechanisms than molecular absorption loss and FSPL, the total LOS loss becomes

$$
\mathrm{PL}(r, f)=\frac{(4 \pi r f)^{2} \exp \left(\kappa_{\mathrm{a}}(f, \mu) r\right)}{c^{2}} G_{\mathrm{Rx}} G_{\mathrm{Tx}},
$$

where $G_{\mathrm{Rx}}$ and $G_{\mathrm{Tx}}$ are the antenna gains and utilizing the polynomial models of this paper, the absorption coefficient $\kappa_{\mathrm{a}}(f, \mu)$ is

$$
\kappa_{\mathrm{a}}(f, \mu)=\sum_{i} y_{i}(f, \mu)+g(f, \mu) .
$$

Fig. 7 shows the levels of the above loss mechanisms assuming unit antenna gains. At low distances, the absorption loss is less significant. The losses around the center of the absorption lines rise to up to about $10 \mathrm{~dB}$ at 10 meter distance, but the impact is very local. However, depending on the application this may be significant, although the loss can be avoided in general by avoiding transmitting on these highloss frequencies. Otherwise the FSPL dominates the results at short distance. At 100 meter distance the molecular absorption becomes more important around the absorption line centers. Therein the losses are increased by few $\mathrm{dBs}$ up to tens of $\mathrm{dBs}$ because of the molecular absorption. If we take the distance to an extreme, considering, e.g., some backhauling applications, the molecular absorption becomes significant and proper signal design (center frequencies, bandwidths, waveforms, etc.) is required. As a rule of thumb, if one considers short range applications, neglecting the molecular absorption does not produce very large error. Only when the distances are increased, the proper modeling of it becomes important. For this purpose, our polynomial model gives very accurate and

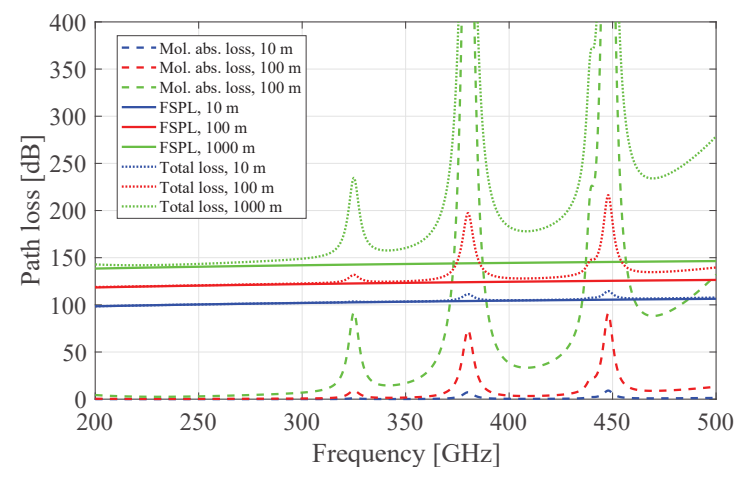

Fig. 7. Molecular absorption loss versus FSPL as a function of frequency for few distances.

simple way to estimate the absorption loss and subsequently the center frequencies, bandwidths, and link budgets of the future systems operating at these high frequency bands.

\section{CONCLUSION}

We derived a simple polynomial model for the molecular absorption loss in the $200-450 \mathrm{GHz}$ band. This model simplifies the estimation of the absorption coefficient as it omits large numbers of tabulated spectroscopic parameters usually required in conjunction with the spectroscopic databases. The model herein depends on the temperature and pressure via their impact on the volume mixing ratio of water vapor in the air. The derived model was shown to be very accurate at all humidity levels. Therefore, this model gives a simple tool to estimate one piece of the total channel response of the future wireless millimeter and sub-millimeter systems.

\section{ACKNOWLEDGEMENT}

This project (TERRANOVA) was supported by Horizon 2020, European Union's Framework Programme for Research and Innovation, under grant agreement no. 761794. This work was also supported in part by the Academy of Finland 6Genesis Flagship under grant no. 318927.

\section{REFERENCES}

[1] T. S. Rappaport et al., "Millimeter wave mobile communications for 5G cellular: It will work!" IEEE Access, vol. 1, no. 1, pp. 335-349, May 2013.

[2] I. Kallfass, F. Boes, T. Messinger, J. Antes, A. Inam, U. Lewark, A. Tessmann, and R. Henneberger, "64 Gbit/s transmission over 850 m fixed wireless link at $240 \mathrm{GHz}$ carrier frequency," J. Infrared Milli. Terahz. Waves, vol. 36, no. 2, pp. 211-233, Feb. 2015.

[3] J. Kokkoniemi, J. Lehtomäki, and M. Juntti, "Simplified molecular absorption loss model for 275 - 400 gigahertz frequency band," in Proc. European Conf. Antennas Propag., 2018, pp. 1-5.

[4] J. M. Jornet and I. F. Akyildiz, "Channel modeling and capacity analysis for electromagnetic nanonetworks in the terahertz band," IEEE Trans. Wireless Commun., vol. 10, no. 10, pp. 3211-3221, Oct. 2011.

[5] L. S. Rothman et al., "The HITRAN 2012 molecular spectroscopic database," J. Quant. Spectrosc. Radiat. Transfer, vol. 130, no. 1, pp. 4-50, Nov. 2013.

[6] ITU-R (2009) Recommendation P.676-8, Attenuation by atmospheric gases, International Telecommunication Union Radiocommunication Sector Std.

[7] S. Paine, "The am atmospheric model," Smithsonian Astrophysical Observatory, Tech. Rep. 152, 2012. 